\title{
Integrals of motion in time periodic Hamiltonian systems: The case of the Mathieu equation
}

\author{
A.C. Tzemos ${ }^{1}$ and G. Contopoulos $2^{2}$ \\ Research Center for Astronomy and Applied Mathematics \\ of the Academy of Athens \\ Soranou Efesiou 4, GR-11527 Athens, Greece
}

\begin{abstract}
We present an algorithm for constructing analytically approximate integrals of motion in simple time periodic Hamiltonians of the form $H=H_{0}+\varepsilon H_{i}$, where $\varepsilon$ is a perturbation parameter. We apply our algorithm in a Hamiltonian system whose dynamics is governed by the Mathieu equation and examine in detail the orbits and their stroboscopic invariant curves for different values of $\varepsilon$. We find the values of $\varepsilon_{\text {crit }}$ beyond which the orbits escape to infinity and construct integrals which are expressed as series in the perturbation $\varepsilon$ and converge up to $\varepsilon_{c r i t}$. In the absence of resonances the invariant curves are concentric ellipses which are approximated very well by our integrals. Finally we construct an integral of motion which describes the hyperbolic stroboscopic invariant curve of a resonant case.
\end{abstract}

\section{Introduction}

The detection of integrals of motion is an important task in the study of dynamical systems. The existence of integrals of motion is related to certain symmetries of the equations of motion of the system (through Noether's theorem) and gives a deep insight into their time evolution. Furthermore it simplifies significantly the calculations, since it decreases the independent variables of the system and can be used for the error control of the calculations.

The integrals of motion appear in two main categories: a) the exact integrals of motion and b) the approximate integrals of motion. In the case

\footnotetext{
${ }^{1}$ thanasistzemos@gmail.com

${ }^{2}$ gcontop@academyofathens.gr
} 
a) these integrals are analytical mathematical expressions of the variables of the system, that do not depend on time. In the case b) the approximate integrals (also called formal integrals) are non-convergent series expansions, truncated at a certain order that remain approximately constant in time.

Formal integrals of motion have been proved to be very useful in Dynamical Astronomy and especially Galactic Dynamics. In particular, their construction in the case of autonomous dynamical systems has already been considered in the past, even to high orders using computer algebra ([1, 2, 3, 4]).

As regards time-periodic Hamiltonians, it was presented back in 1966 for the first time a general method for the calculation of formal integrals [5]. This method was applied to some simple Hamiltonians of one and two degrees of freedom. However, due to the lack of computer algebra systems at that time, the calculations were made by hand and were limited to second order with respect o the perturbation parameter $\varepsilon$.

In recent years there has been much interest in periodic in time Hamiltonians, especially in Russia. Most of this work dealt with stability problems in one or more degrees of freedom (e.g. Markeev [6, 7, 8, 9, 10], Kholostova [11, 12, 13], Bardin and Lanchares [14]). More recently Bruno ([15, 16]) calculated normal forms in particular problems.

On the other hand some authors (Kandrup [17, 18] and Terzić and Kandrup [19]) have calculated orbit in time-periodic potentials, with emphasis on the generation of chaos.

Our interest in this problem was revived because of our need to compare the classical with the quantum mechanical results (Efthymiopoulos and Contopoulos [20]).

In the present paper we exploit the power of the Maple Computer Algebra System in order to construct high order integrals of motion in simple time periodic Hamiltonian systems. As a first step towards this direction we apply our algorithm on a simple Hamiltonian system whose equations of motion can be written in a single second order differential equation of the form:

$$
\frac{d^{2} x}{d t^{\prime 2}}+\left[a-2 q \cos \left(2 t^{\prime}\right)\right] x=0
$$

Although this differential equation is linear in $\mathrm{x}$ and there is no chaos in its orbits, it is very useful in Applied Mathematics. It is the well known Mathieu equation (ME) [21, 22] and has applications in various fields of physical sciences, such as Acoustics (e.g. in the study of an elliptical drum [23]), Quantum Mechanics (e.g. in the study of the quantum pendulum [24]), 
General Relativity (e.g. in the study of the solutions of wave equations in curved spaces [25]) and Quantum Chemistry (charged particle in a quadruple field [26]). Many applications of MEs can be found in [27].

As already known from the theory, different parameters in ME can lead to bounded or unbounded motion. We make a detailed study of the orbits and their invariant curves on a stroboscopic surface of section by solving numerically in Python 3.7 the Hamilton equations. In the case of bounded orbits we construct in Maple 2016 an integral of motion which is convergent for small perturbations and describes very well the invariant curves. This integral becomes divergent and the forms of the orbits change abruptly at the threshold of the escapes.

In section 2 of the present paper we give the Hamiltonian of our model and describe our algorithm for the calculation of formal integrals of motion up to an arbitrary order in the absence of resonances. Then in Section 3 we present our results in the case where the perturbation parameter $\varepsilon$ is positive, by calculating both the orbits and their stroboscopic sections (i.e. the distribution of their points after successive periods). We apply our algorithm and calculate the integrals at successive orders of the perturbation parameter and show their convergence to the form of the invariant curves on the stroboscopic sections. Then in Section 4 we examine the case of the negative values of the perturbation parameter and in Section 5 we study a formal integral in a resonant case. Finally in Section 6 we summarize our results and draw our conclusion.

\section{Integral of the Mathieu equation}

In the special case of the Mathieu equation we have

$$
H=H_{0}+\varepsilon H_{i}=\frac{1}{2}\left(y^{2}+\omega_{i}^{2} x^{2}\right)-\varepsilon x^{2} \cos (\omega t)
$$

The corresponding equations of motion are:

$$
\frac{d x}{d t}=y, \quad \frac{d y}{d t}=\frac{d^{2} x}{d t^{2}}=-\left[\omega_{1}^{2}-2 \varepsilon \cos (\omega t)\right] x
$$

The second equation takes the form of the Mathieu equation if we set $\omega t=$ $2 t^{\prime}, a=4 \omega_{1}^{2} / \omega^{2}$ and $q=\frac{4 \varepsilon}{\omega^{2}}$. In particular if $\omega=2$ we have $t=t^{\prime}, a=\omega_{1}^{2}$ and 
$q=\varepsilon$. There are resonance conditions when $a=1,4,9,16 \ldots$ For $\omega=2$ resonances appear if $\omega_{1}=1,2,3, \ldots$ An integral of motion

$$
\Phi=\Phi_{0}+\varepsilon \Phi_{1}+\varepsilon^{2} \Phi_{2}+\cdots+\varepsilon^{s} \Phi_{S}+\ldots
$$

must satisfy the equation

$$
\frac{d \Phi}{d t}=\frac{\partial \Phi}{\partial t}+[\Phi, H]=0
$$

where $[\Phi, H]$ is the Poisson bracket

$$
[\Phi, H] \equiv \frac{\partial \Phi}{\partial x} \frac{\partial H}{\partial y}-\frac{\partial \Phi}{\partial y} \frac{\partial H}{\partial x}
$$

We apply Eq.(3) to the terms of successive orders in $\varepsilon$ and find

$$
\frac{\partial \Phi_{s+1}}{\partial t}+\left[\Phi_{s+1}, H_{0}\right]-K_{s}=0
$$

where

$$
K_{s}=-\left[\Phi_{s}, H_{1}\right]
$$

If we set $\Phi_{0}=H_{0}$ we can calculate successively the terms of various orders of $\Phi$ expressed in trigonometric terms of multiplicities of $\omega t$. From the characteristic curves of Eq. (7) we find

$$
d t=\frac{d x}{y}=\frac{d y}{-\omega_{1}^{2} x}=\frac{d \Phi_{s+1}}{K_{s}}
$$

The zero order solution is

$$
x=\frac{\sqrt{2 \Phi_{0}}}{\omega_{1}} \sin \left(\omega_{1} t\right), y=\sqrt{2 \Phi_{0}} \cos \left(\omega_{1} t\right)
$$

Consequently from Eqs. (9) we get

$$
\Phi_{s+1}=\int_{0}^{t} K_{s} d t
$$

where $K_{S}$ is expressed in trigonometric terms of multiples of $\omega t$ and $\omega_{1} t$. After the integration we use again Eqs. (10) to express back the trigonometric terms 
of $\Phi_{s+1}$ which contain $\omega_{1} t$ in terms of $y^{2}, x^{2}, x y$ multiplied by trigonometric terms of multiples of $\omega t$. Consequently the integral $\Phi$, which is in practice truncated at some order, is $\Phi=\Phi(x, y, \omega t)$.

In particular $\Phi_{0}=H_{0}$ and

$$
\Phi_{1}=\int_{0}^{t} \frac{\partial \Phi_{0}}{\partial y} \frac{\partial H_{1}}{\partial x} d t=-2 \int_{0}^{t} x y \cos (\omega t) d t
$$

The integral $\Phi$ is a series in $\varepsilon$ and of second order in $x, y$. Up to second order in $\varepsilon$ it is:

$$
\Phi=\frac{1}{2}\left(C_{x} \omega_{1}^{2} x^{2}+C_{y} y^{2}+C_{x y} x y\right)
$$

where

$$
\begin{aligned}
C_{x}=1 & +\frac{4 \varepsilon(\cos (\omega t)+1)}{\omega^{2}-4 \omega_{1}^{2}} \\
& +\frac{\varepsilon^{2}\left(16\left(\omega^{2}-\omega_{1}^{2}\right) \cos (\omega t)+\left(4 \omega_{1}^{2}+7 \omega^{2}-\frac{2 \omega^{4}}{\omega_{1}^{2}}\right) \cos (2 \omega t)+12 \omega_{1}^{2}-7 \omega^{2}+\frac{4 \omega^{4}}{\omega_{1}^{2}}\right)}{\left(\omega^{2}-4 \omega_{1}^{2}\right)^{2}\left(\omega^{2}-\omega_{1}^{2}\right)} \\
& +\ldots \\
C_{y}=1 & -\frac{4 \varepsilon(\cos (\omega t)-1)}{\omega^{2}-4 \omega_{1}^{2}} \\
& +\frac{\varepsilon^{2}\left(-16\left(\omega^{2}-\omega_{1}^{2}\right) \cos (\omega t)+3\left(\omega^{2}-4 \omega_{1}^{2}\right) \cos (2 \omega t)+13 \omega^{2}-4 \omega_{1}^{2}\right)}{\left(\omega^{2}-4 \omega_{1}^{2}\right)^{2}\left(\omega^{2}-\omega_{1}^{2}\right)}+\ldots
\end{aligned}
$$

and

$C_{x y}=\frac{-4 \varepsilon \omega \sin (\omega t)}{\omega^{2}-4 \omega_{1}^{2}}+\frac{\varepsilon^{2} \omega\left(-16\left(\omega^{2}-\omega_{1}^{2}\right) \sin (\omega t)+6\left(\omega^{2}-4 \omega_{1}^{2}\right) \sin (2 \omega t)\right)}{\left(\omega^{2}-4 \omega_{1}^{2}\right)^{2}\left(\omega^{2}-\omega_{1}^{2}\right)}+\ldots$

We notice that the first order terms in $\varepsilon$ contain $\cos (\omega t), \sin (\omega t)$ and a constant, the second order terms contain also $\cos (2 \omega t)$ and $\sin (2 \omega t)$ and so on. The constant terms are due to the fact that the integral starts at $t=0$. 


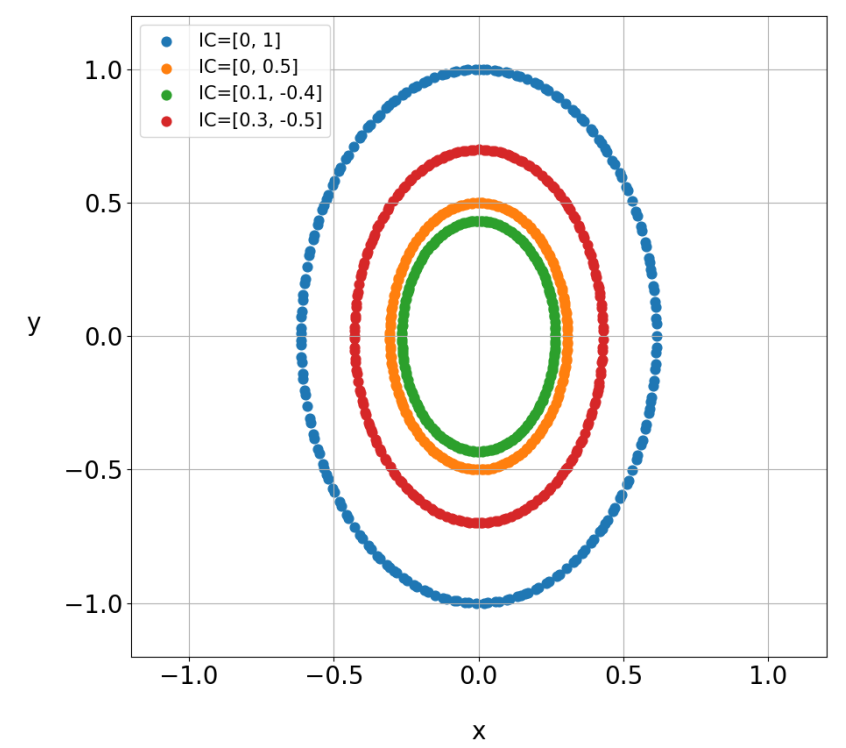

Figure 1: The invariant curves (ellipses) of the stroboscopic surface of section in the case $\omega=2, \omega_{1}=0.9, \varepsilon=0.1$ and various initial conditions (IC) for the first 200 periods. The various concentric ellipses are similar to each other.

In general the terms of order $\varepsilon^{m}$ contain cosines of multiples of $\omega t$ up to order $m \omega t$ together with constant terms in the coefficients of $x^{2}$ and $y^{2}$, while they contain only sines up to order $m \omega t$ in the coefficient of $x y$. The denominators contain factors of the form $\left(\omega^{2}-4 \omega_{1}^{2}\right),\left(\omega^{2}-\omega_{1}^{2}\right),\left(\omega^{2}-9 \omega_{1}^{2}\right), \ldots\left(\omega^{2}-\right.$ $\left.m^{2} \omega_{1}^{2}\right)$.

Using our program for the construction of this integral we have calculated its terms up to order 28 . For $\varepsilon$ not large the integral $\Phi$ represents an ellipse of the form

$$
\Phi=A x^{2}+B y^{2}+2 D x y
$$

If $t=2 k \pi / \omega=k T$ we have

$$
A=\frac{\omega_{i}^{2}}{2}\left[1+\frac{8 \varepsilon}{\omega^{2}-4 \omega_{1}^{2}}+\frac{2 \varepsilon^{2} \omega^{2}\left(\omega^{2}+8 \omega_{1}^{2}\right)+\ldots}{\omega_{1}^{2}\left(\omega^{2}-4 \omega_{1}^{2}\right)^{2}\left(\omega^{2}-\omega_{1}^{2}\right)}\right], \quad B=\frac{1}{2}, D=0
$$

This represents an ellipse passing through the initial point $\left(x_{0}=0, y_{0}=\right.$ 1) with semiaxes $a=\sqrt{\frac{\Phi}{A}}, b=\sqrt{\frac{\Phi}{B}}$. The points $(x, y)$ of an orbit on a 
stroboscopic Poincaré surface of section $t=k T$ lie on this ellipse, if the series giving $A$ converges.

We have calculated several orbits and verified that if $\varepsilon$ is small and $\omega$ is not close to a resonance $\omega=\omega_{i}, 2 \omega_{i}, 3 \omega_{i} \ldots$ the points of the orbits on the stroboscopic surface of sections lie on such an ellipse. Figure 1 represents a set of such ellipses for $\varepsilon=0.1, \omega=2$ and $\omega_{1}=0.9$. In fact for various initial conditions we have similar concentric ellipses.

\section{Applications for $\varepsilon>0$}

If we fix $\omega=2$ and $\omega_{1}=0.9$, and initial condition $x_{0}=0, y_{0}=1$ we have $\Phi=1 / 2$. Then the semiaxes of the ellipses are $a=\frac{1}{\sqrt{2 A}}, b=1$. In Fig. 2 we present the stroboscopic surface of section for these parameters for varlous values of $\varepsilon$. We observe that they are all ellipses with one axis from $y=1$ to $y=-1$. The same holds for other non-resonant values of $\omega$ and $\omega_{1}$.

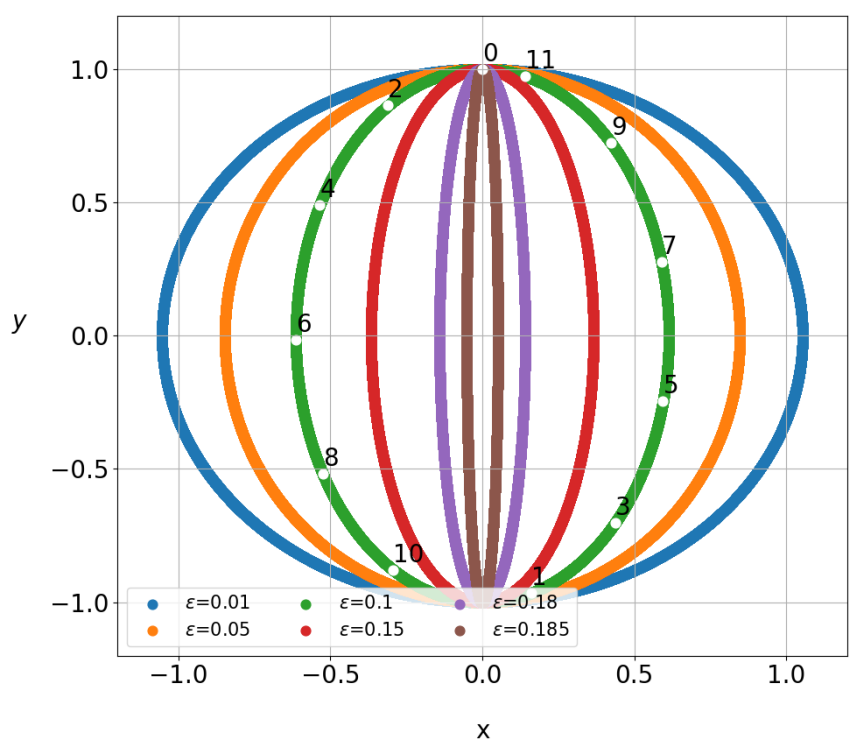

Figure 2: Invariant curves on the stroboscopic surface of section in the case with $\omega=2, \omega_{1}=0.9$ for various values of $\varepsilon$ and the same initial conditions $\left(x_{0}=0, y_{0}=1\right)$. In particular we have given the successive points $1,2, \ldots, 11$ covering clockwise the invariant curve for $\varepsilon=0.1$. 

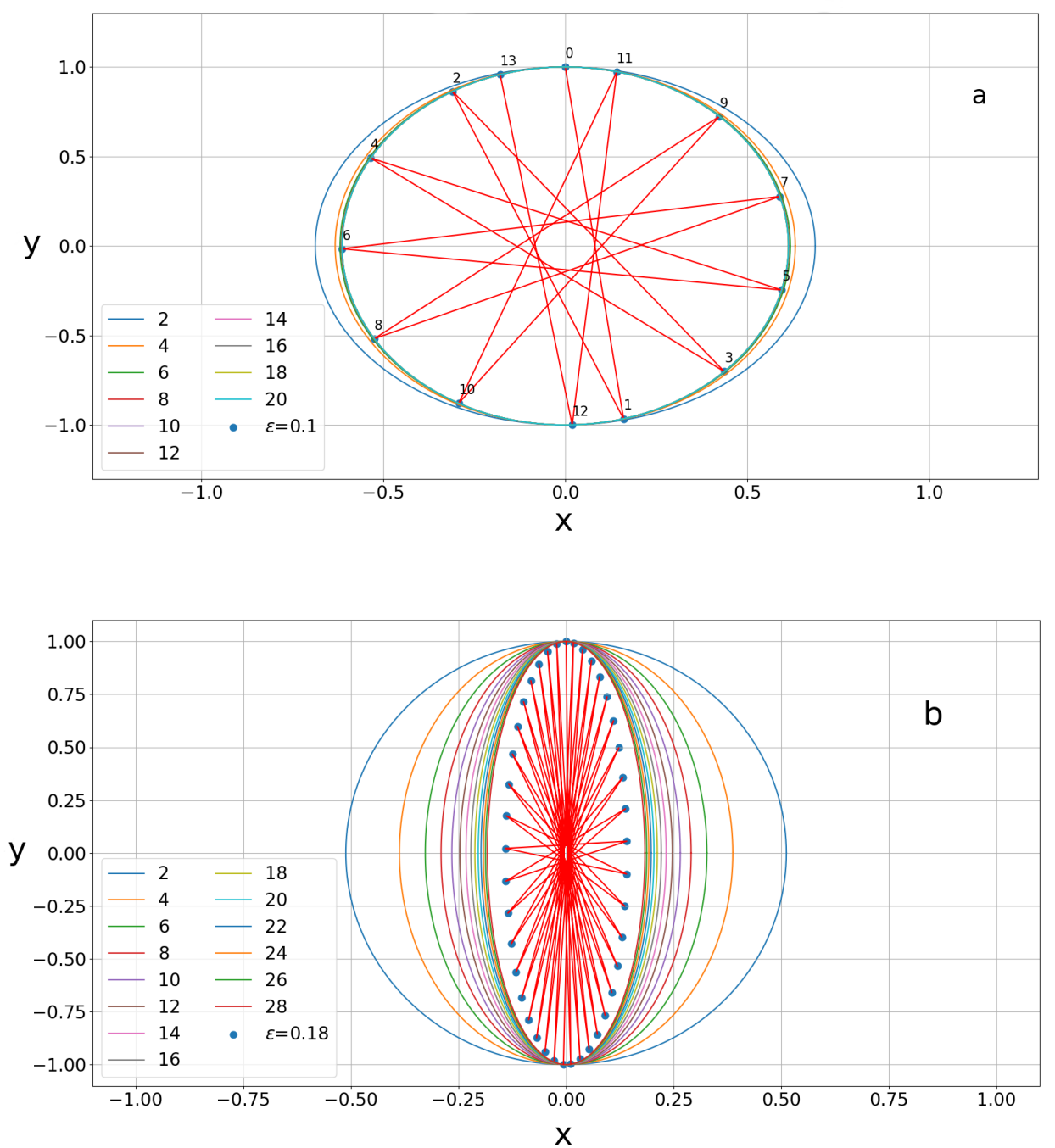

Figure 3: Successive points of the orbits with $\omega=2, \omega_{1}=0.9$ and initial conditions $\left(x_{0}=0, y_{0}=1\right)$ on the stroboscopic surface of section in the cases (a) $\varepsilon=0.1$ and (b) $\varepsilon=0.18$. The successive points are joined by straight lines. The points cover roughly for the first time the corresponding invariant curve. Their numbers are 13 in case (a) and 39 in case (b). The set of points is approached by the theoretical invariant curves truncated at various successive orders as shown by colors. 
In Figs. 3a,b we mark the successive points after times $T, 2 T, 3 T \ldots$ etc. for $\varepsilon=0.1$ and $\varepsilon=0.18$. At the same time we draw the ellipses found if we truncate the integral $\Phi$ after the terms of order $2,4,6 \ldots$ in $\varepsilon$. We see that the ellipse of order 2 is far from the points of the numerical solution. But as we increase the order of the truncation the ellipses approach gradually the invariant curve formed by the successive points. In the case $\varepsilon=0.1$ we have good convergence at order 6 , in the case $\varepsilon=0.15$ good convergence is reached at order 20 , while in the case $\varepsilon=0.18$ we have not yet reached good convergence up to order 28. However these figures indicate that the integral $\Phi$ in fact converges all the way up to $\varepsilon=0.1857$, namely up to the critical value of $\varepsilon$ above which we have escapes to infinity.

Moreover we see that the angles between the lines joining the successive points decrease as $\varepsilon$ increases. In particular for $\varepsilon=0$ the successive points are on a circle on a plane $\left(\omega_{1} x, y\right)$ and the successive angles are $\Delta \phi=\frac{2 \pi \omega_{1}}{\omega}=0.9 \pi$. Thus the number of points required to cover roughly the invariant curve is about 11 (Fig. 3 a). In the case $\varepsilon=0.1$ this number is about 13 , for $\varepsilon=0.15$ it is 17 , for $\varepsilon=0.18$ it is about 40 and for $\varepsilon=0.185$ it is almost 110 points. The following iterations give additional points that fill more densely the ellipse provided by the integral $\Phi$.

These numbers are found also by calculating the distances $d=\sqrt{\omega_{1}^{2} x^{2}+y^{2}}$ of the successive points on the stroboscopic surface of section as functions of time (Fig. 4). All the orbits start at $\left(x_{0}=0, y_{0}=1\right)$ with $d=1$ and decrease down to a minimum distance. Then they increase up to the distance $d=1$ and continue to decrease and increase. We see that for $\varepsilon=0.01$ one comes back to the maximum distance after 11 points, for $\varepsilon=0.1$ after 13 points, and so on. Figure 4 gives also the time required for the successive points to reach again the maximum distance. This time increases considerably as $\varepsilon$ approaches the critical value $\varepsilon_{\text {crit }}=0.1857$. 


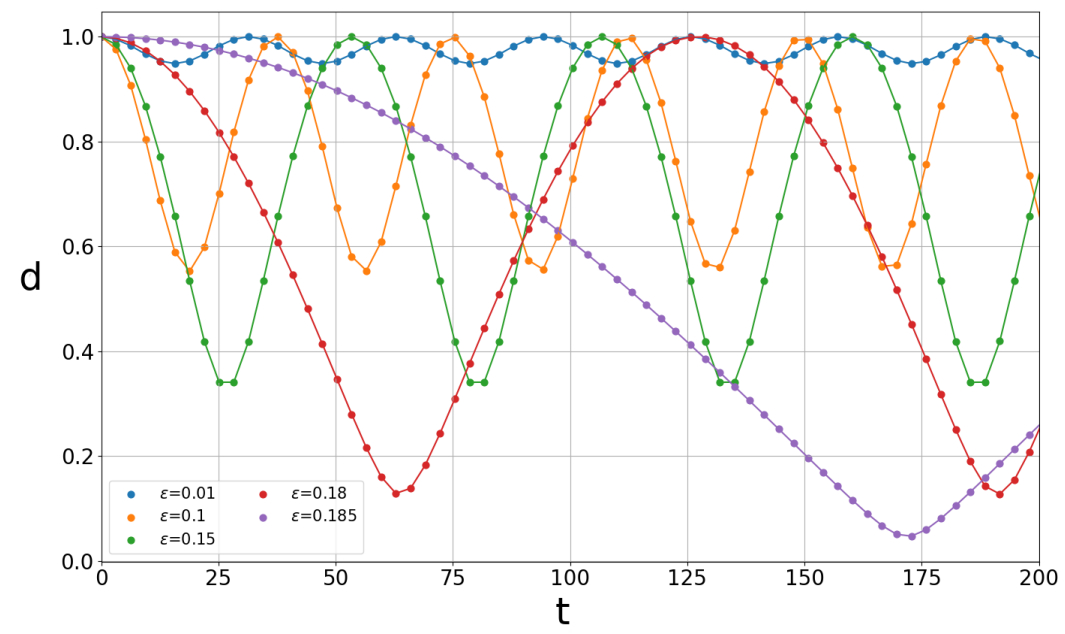

Figure 4: The distances $d \equiv \sqrt{\omega_{1}^{2} x^{2}+y^{2}}$ between the origin $(0,0)$ and the successive points of the intersections of the orbits by the stroboscopic surface of section, for various values of $\varepsilon$ up to $t=200$. We have $\omega=2, \omega_{1}=0.9, x_{0}=$ $0, y_{0}=1$.

The motion with initial conditions $\left(x_{0}=0, y_{0}=1\right)$ for $\varepsilon<\varepsilon_{\text {crit }}$ starts by spiralling inwards until it reaches a minimum distance and then it spirals outwards and so on. Thus the orbit fills a ring as in Fig.5a for $\varepsilon=0.1$ and its corresponding invariant curve is an ellipse. As $\varepsilon$ increases the ring becomes broader and the empty hole near the center becomes smaller as in Fig. 5b for $\varepsilon=0.185$. Then the invariant ellipse becomes very thin and in the limit $\varepsilon \rightarrow \varepsilon_{\text {crit }}$ the ellipse tends to the straight line from $y=1$ to $y=-1$ while the empty region vanishes. 

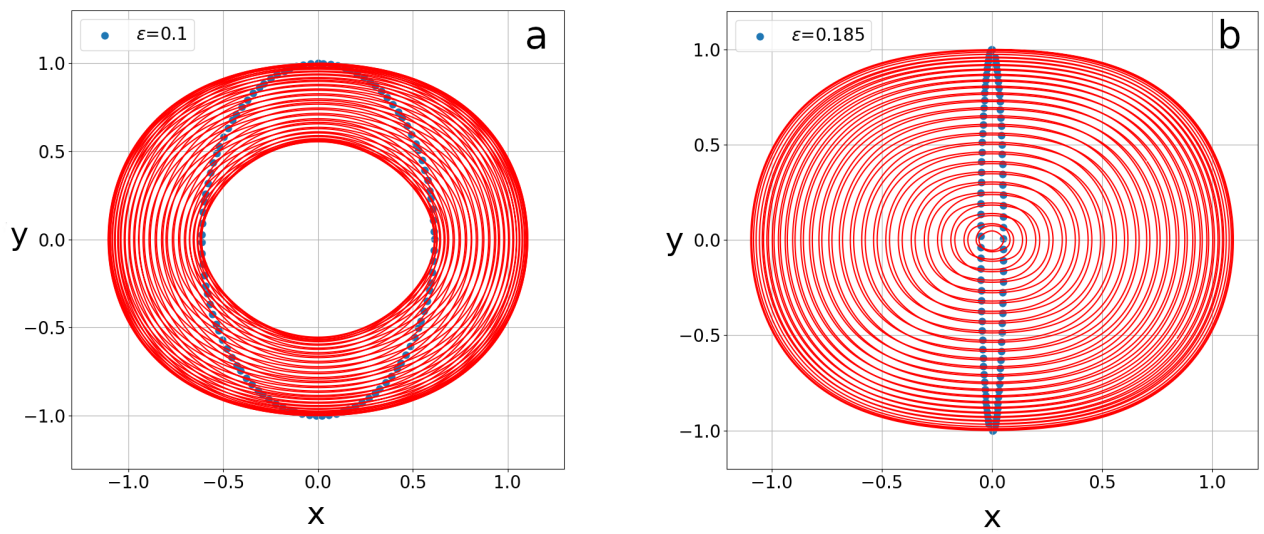

Figure 5: Orbits in the case $\omega=2, \omega_{1}=0.9$ together with their intersections by the stroboscopic surface of section. (a) For $\varepsilon=0.1$ and 106 periods. This orbit fills a ring inside the original arc of the orbit starting at $\left(x_{0}=0, y_{0}=1\right)$. (b) For $\varepsilon=0.185$ and 100 periods. This orbit fills a ring leaving only a small hole around the center $(0,0)$.

For particular values of $\varepsilon$ the orbit comes exactly to its initial point $x_{0}=$ $0, y_{0}=1$ after a number $n$ of periods i.e. we have a periodic orbit of period $n T$. Such is the case of Fig. 6 for $\varepsilon=0.15$, where we have a periodic orbit of period $17 T$. In this case the energy $E$ increases to a maximum, but then it returns to its initial value. Similar periodic orbits of other periods appear for other values of $\varepsilon$.

If $\varepsilon$ goes beyond a critical value about $\varepsilon_{\text {crit }}=0.1857$ the orbits escape to infinity. In the present study we notice that the series $\Phi$ representing the integral of motion does not converge beyond the critical value of $\varepsilon$. E.g. for $\varepsilon=0.19$ (Fig,7) the orbit starts close to an initial ellipse but then makes a spiral outwards that extends to about $x= \pm 15, y= \pm 15$ after 50 periods. In Fig. 8 we see that the logarithms of the distances for particular values of $\varepsilon$ beyond the critical value increase linearly in time. Therefore the distances of the escaping orbits increase exponentially in time and the increase is larger for larger $\varepsilon$. We know from the theory that this motion extends to infinity. 


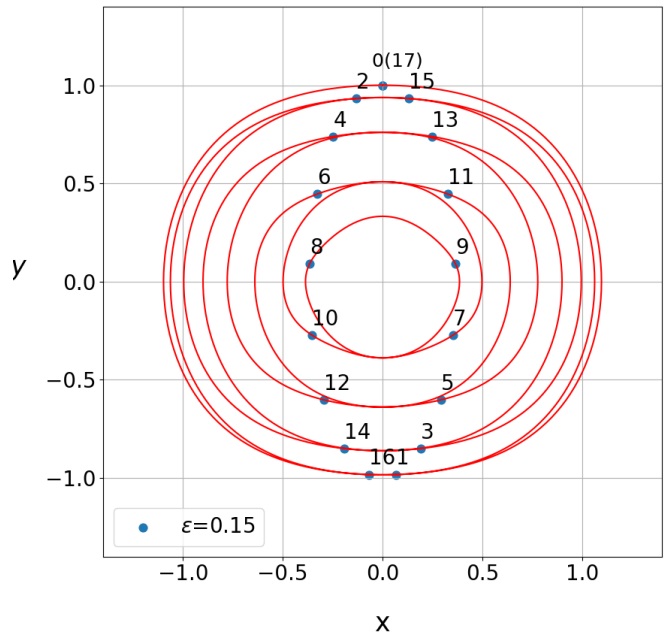

Figure 6: A periodic orbit of period $17 T$ where $T=2 \pi / \omega$, together with the 17 points of intersection with the stroboscopic surface of section.

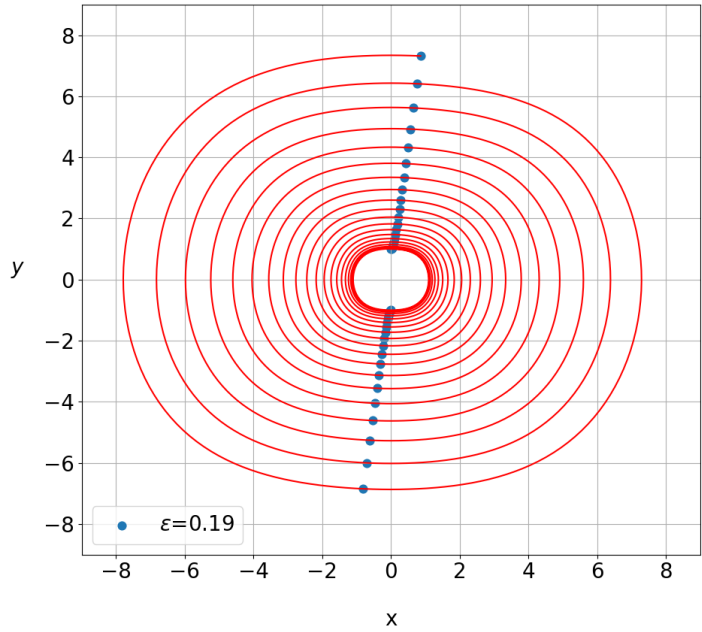

Figure 7: An escaping orbit for $\left(\omega=2, \omega_{1}=0.9, \varepsilon=0.19\right)$ starting at the point $\left(x_{0}=0, y_{0}=1\right)$ for the first 40 periods, together with its intersections with the stroboscopic surface of section. 


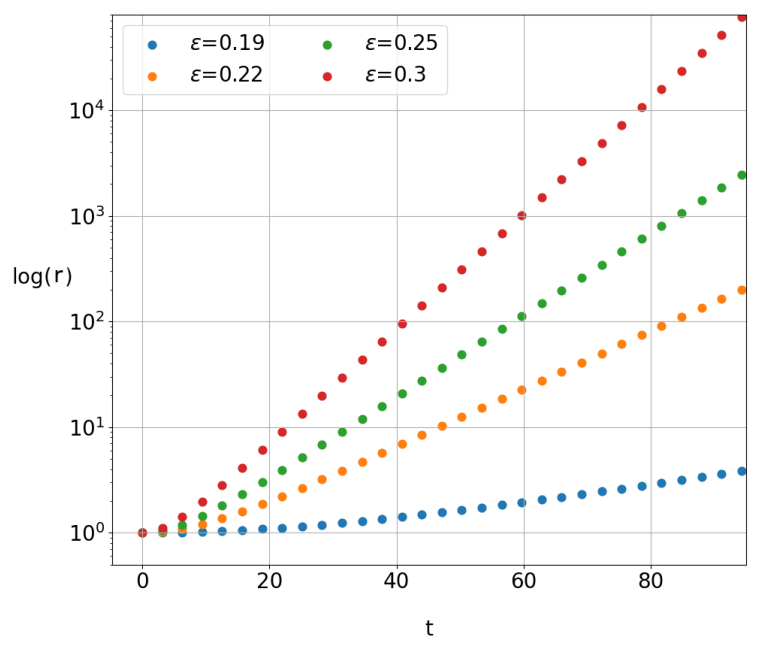

Figure 8: The logarithms of the distances $r=\sqrt{x^{2}+y^{2}}$ of the successive intersections of escaping orbits by the stroboscopic surface of section for $\omega=2, \omega_{1}=0.9, x_{0}=0, y_{0}=1$ and various values of $\varepsilon$ up to $t=30 T$. In all these cases the logarithms of the distances increase linearly in time thus the distances icnrease exponentially in time. The distances are longer for larger $\varepsilon$.

An interesting aspect of our problem is found if we work in the so called extended phase space where our system becomes conservative. Namely we extend the phase space to include the ordinary time as a canonical variable and its conjugate momentum $E$ which is minus the energy. Then the new Hamiltonian

$$
\bar{H}(x, y, E, t)=H(x, y, t)+E
$$

is conservative with respect to a fictitious time variable $\tau=t$ and the Hamilton equations read:

$$
\begin{aligned}
& \frac{d x}{d t}=\frac{\partial H}{\partial y}, \quad \frac{d y}{d t}=-\frac{\partial H}{\partial x} \\
& \frac{d \tau}{d t}=\frac{\partial \bar{H}}{\partial E}=1, \quad \frac{d E}{d t}=-\frac{\partial \bar{H}}{\partial t}=-\frac{\partial H}{\partial t}
\end{aligned}
$$

Then we can calculate the value of the energy $E$ as a function of time using the last Eq. (20) and we find that the value of $\bar{H}$ is very close to zero 
(in fact it is of order $\mathcal{O}\left(10^{-8}\right)$ which is the accuracy of our calculations). In the cases $0<\varepsilon<\varepsilon_{\text {crit }}$ for $x_{0}=0, y_{0}=1$ the value of $E$ starts at $E=-0.5$ for $t=0$, reaches a maximum near $E=0$ and then oscillates between this maximum and a minimum. The values of $x$ and $E$ of the stroboscopic section are given by blue dots (Fig. 9a). These dots mark the points of the invariant curve on the stroboscopic section. The first 40 points are successively on the right and on the left part of the hyperbolic-like invariant curve which is directed upwards. Further points fill this curve densely. On the other hand for $\varepsilon>\varepsilon_{\text {crit }}$ the value of $E$ decreases continuously on the average (Fig. $9 \mathrm{~b}$ ) and tends to $-\infty$. The dots are again on a hyperbola-like curve, but this time the curve is directed downwards and has no limit. This difference allows us to find with great accuracy the transition value $\varepsilon_{\text {crit }}$ for the escapes. At $\varepsilon=\varepsilon_{\text {crit }} \simeq 0.1857848626$ the orbit is periodic and it is represented by the point $\left(x=x_{0}=0, y=y_{0}=1\right)$ on the stroboscopic surface of section. We have calculated the monodromy matrix of this periodic orbit and found that its eigenvalues are all equal to one. Therefore the orbit is marginally unstable.
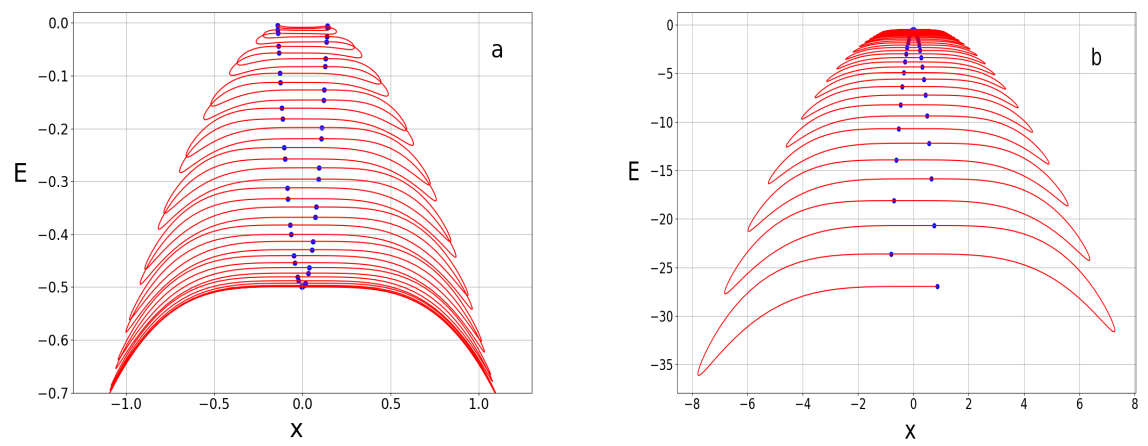

Figure 9: Stroboscopic surfaces of section (blue points). The trajectory of $E$ as a function of $x$ for (a) $\varepsilon=0.18$ up to 41 periods and (b) for $\varepsilon=0.19$ up to 40 periods. We observe that in the case (a) the trajectory is confined and the blue points oscillate in a certain range of energies, while in the case (b) the trajectory escapes to $-\infty$ and the blue points also tend to $-\infty$. In the extended space, the total energy $\bar{H}=H+E$ is conserved and equal to 0 for our parameters.

In the extended phase space we can calculate another integral

$$
\Psi=\Psi_{0}+\varepsilon \Psi_{1}+\varepsilon^{2} \Psi_{2}+\ldots
$$


if we set $\Psi_{0}=E$. Then we find

$$
\Psi_{1}=\frac{\left[\left(2 \omega_{1}{ }^{2}-\omega^{2}\right) x^{2}+2 y^{2}\right] \cos (\omega t)+2 \sin (\omega t) \omega x y-2\left(\omega_{1}^{2} x^{2}+y^{2}\right)}{\omega^{2}-4 \omega_{1}^{2}}
$$

But then we notice that

$$
\Phi_{1}+\Psi_{1}=-x^{2} \cos (\omega t)=H_{1},
$$

therefore

$$
\Psi_{1}=H_{1}-\Phi_{1}
$$

If we calculate now $\Psi_{2}$ this is equal to $-\Phi_{2}$ because $\left[H_{1}, H_{1}\right]=0$. In the same way we find $\Psi_{3}=-\Phi_{3}$ and so on. Thus

$$
\Phi+\Psi=\frac{1}{2}\left(\omega_{1}^{2} x^{2}+y^{2}\right)+E-\varepsilon x^{2} \cos (\omega t)=\bar{H}=0 .
$$

Consequently we have only two independent isolating integrals $\bar{H}$ and $\Phi$, thus the transformation of the system to an autonomous Hamiltonian system does not give any new results.

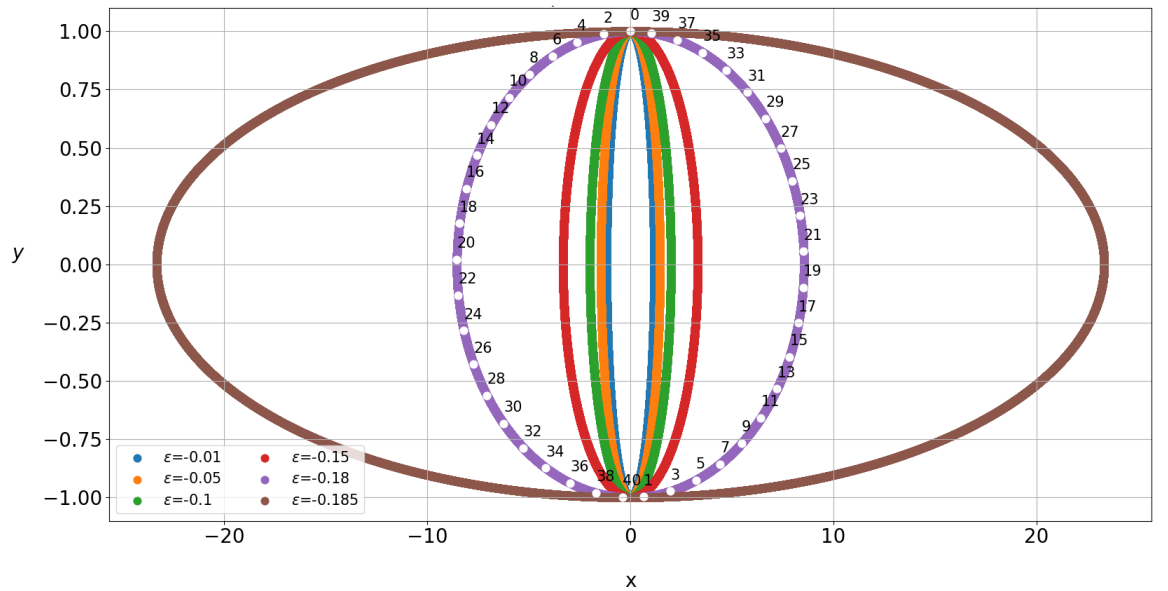

Figure 10: Invariant curves on a stroboscopic surface of section in the case $\omega=2, \omega_{1}=0.9$ for $x_{0}=0, y_{0}=1$ and various negative values of $\varepsilon$. As $\varepsilon$ decreases and approaches the critical value $\varepsilon_{\text {crit }} \simeq-0.1857$ the size of the curve along $x$ increases and tends to $\infty$. In the case $\varepsilon=-0.18$ we give also the successive points corresponding to successive periods. 

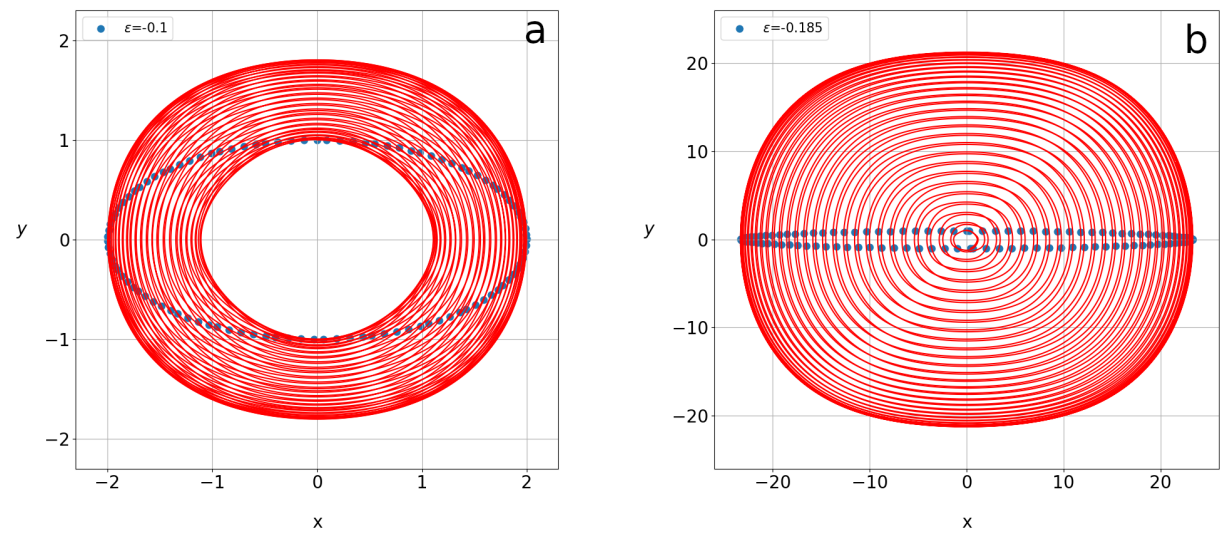

Figure 11: Orbits in the case $\omega=2, \omega_{1}=0.9$ (a) For $\varepsilon=-0.1$ and 106 periods. The orbit fills a ring outside the original arc of the orbit starting at $\left(x_{0}=0, y_{0}=1\right)(\mathrm{b})$ For $\varepsilon=-0.185$ and 110 periods. We observe that the orbit extends to much larger distances than in the case (a).

\section{Cases with $\varepsilon<0$}

If $\varepsilon<0$ we have again ellipses on the stroboscopic Poincaré surface of section. However these ellipses are outside the ellipse for $\varepsilon=0$ (Fig. 10). The critical value of $\varepsilon$ is $\varepsilon_{\text {crit }}=-0.1857$, symmetric to the critical value $\varepsilon_{\text {crit }}=0.1857$ for positive $\varepsilon$. The stroboscopic ellipses become longer along the $\mathrm{x}$-axis as $\varepsilon$ increases and they tend to infinity as $\varepsilon$ tends to the critical value.

The orbits for $\varepsilon<0$ are different from those of $\varepsilon>0$. The orbits are outside the limiting ellipse $\omega_{1}^{2} x^{2}+y^{2}=1$ for $\varepsilon=0$. The number of points on the stroboscopic ellipse required to cover once the whole ellipse again increases as $|\varepsilon|$ increases. For example for $\varepsilon=-0.1$ this number is 39 (Fig. 10 and for $\varepsilon=-0.185$ it is $n=110$ (Figs 11a,b). The orbits for $\varepsilon<0$ extend to large distances both in $x$ and $y$ but with much smaller ellipticity than the corresponding stroboscopic ellipse. Finally for $|\varepsilon|$ larger than the critical value $\left|\varepsilon_{\text {crit }}\right|=0.1857$ the orbits escape again to infinity. The form of such an orbit is shown in Fig. 12 for $\varepsilon=-0.19$.

If we change now the value of $\omega_{1}$ we find again ellipses for various values of $\varepsilon$ given by the integral of motion. However the critical values of $\varepsilon$ are now different. We consider here two examples. 


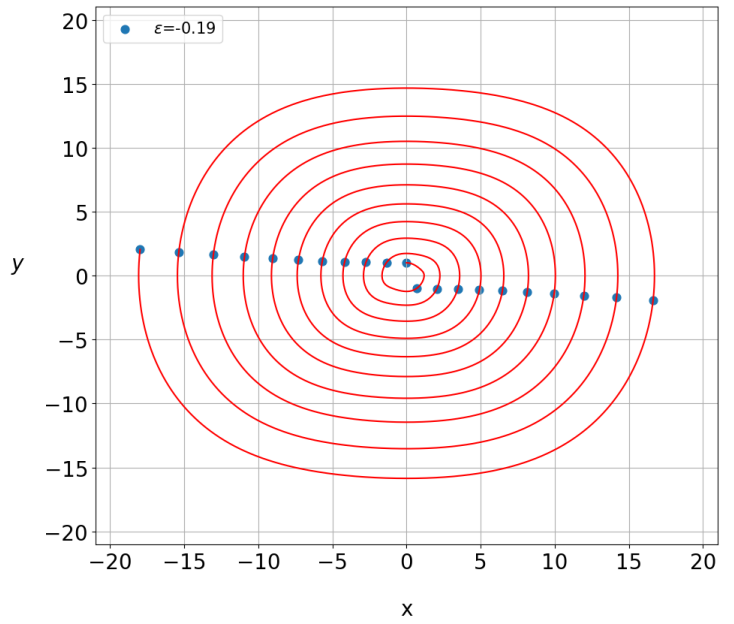

Figure 12: An escaping orbit for $\omega=2, \omega_{1}=0.9, x_{0}=0, y_{0}=1, \varepsilon=-0.19$ for the first 20 periods, together with its intersections with the stroboscopic surface of section.

1. In the case $\omega_{1}=0.1$ we have the ellipses of Fig. 13. In this case the limiting ellipse $\omega_{1}^{2} x^{2}+y^{2}=1$ is just outside the ellipse for $\varepsilon=0.001$, i.e. it is much more elongated along the $\mathrm{x}$-axis than in the case with $\omega_{1}=0.9$. Furthermore the successive points on the stroboscopic surface of section are much closer to each other. The total number of points to cover roughly the ellipse once for $\varepsilon=0.1$ is $N \simeq 16$. As $\varepsilon$ increases this number increases. In this case the critical value of $\varepsilon$ is $\varepsilon_{\text {crit }}=0.89964$, i.e. much larger that in the case $\omega_{1}=0.9$.

2. The second case is $\omega_{1}=1.1$, larger than the resonant value $\omega_{1}=1$. In this case the critical value of $\varepsilon$ is about $\varepsilon_{\text {crit }}=0.21598$. We have again ellipses on the stroboscopic surface of section for $\varepsilon<\varepsilon_{\text {crit }}$ but these become larger as $\varepsilon$ increases (Fig. 14a). The difference between the behaviour of the case $\omega_{1}=1.1$ and the cases $\omega_{1}=0.9$ and $\omega_{1}=0.1$ can be explained theoretically. In fact close to $\varepsilon=0$ the value of the coefficient $A$ of Eq. (18) is approximately

$$
A \simeq \frac{\omega_{1}^{2}}{2}\left(1+\frac{8 \varepsilon}{\omega^{2}-4 \omega_{1}^{2}}\right)
$$


while $\Phi=1 / 2$ for $\left(x_{0}=0, y_{0}=1\right)$ hence

$$
a \simeq \frac{1}{\omega_{1}}\left(1-\frac{4 \varepsilon}{\omega^{2}-4 \omega_{1}^{2}}\right)
$$

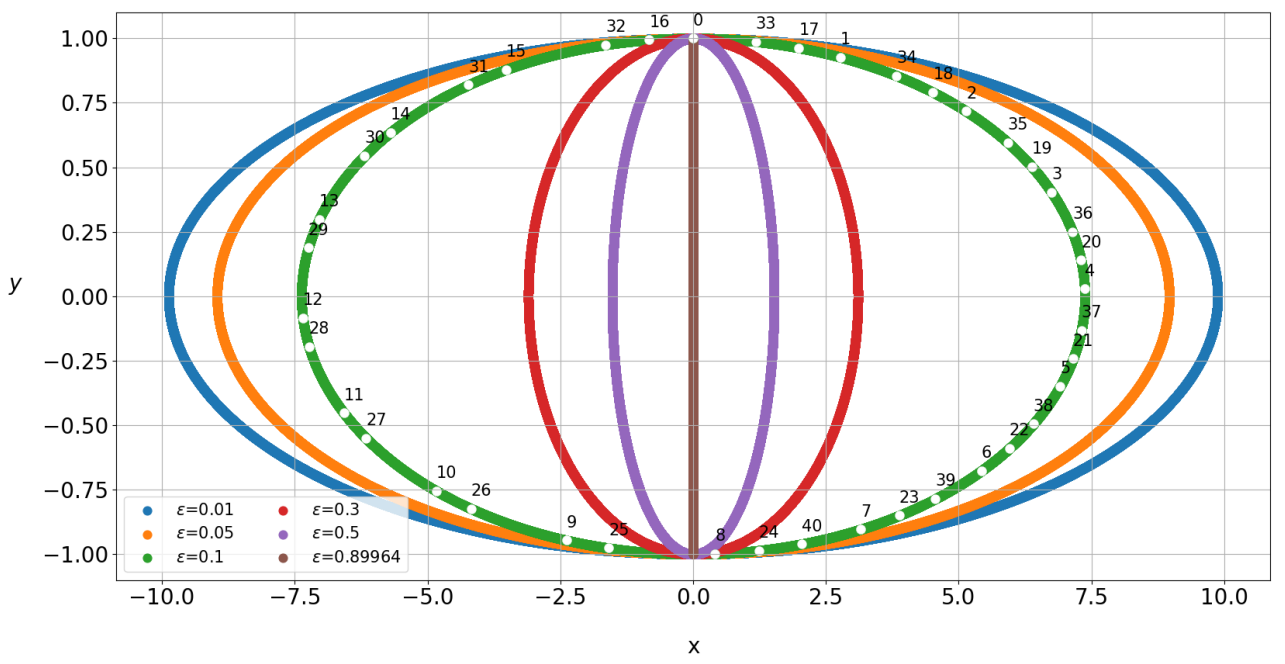

Figure 13: Invariant curves (ellipses) on the stroboscopic surface of section on the case $\omega=2, \omega_{1}=0.1, x_{0}=0, y_{0}=1$, for various values of $\varepsilon>0$. As $\varepsilon$ increases the invariant curves shrink and for $\varepsilon=0.89964$ the curve is very elongated close to a critical value (almost a straight line). In the case $\varepsilon=0.1$ we give also the successive points.

Therefore for $\varepsilon>0$ and $\left(\omega^{2}-4 \omega_{1}^{2}\right)>0$ we have $\varepsilon /\left(\omega^{2}-4 \omega_{1}^{2}\right)>0$, hence $a<\frac{1}{\omega_{1}}$, i.e. the ellipses are inside the ellipse for $\varepsilon=0$, while for $\varepsilon<0$ and $\left(\omega^{2}-4 \omega_{1}^{2}\right)>0$ we have $a>\frac{1}{\omega_{1}}$, i.e. the ellipses are outside the ellipse for $\varepsilon=0$. On the other hand for $\left(\omega^{2}-4 \omega_{1}^{2}\right)<0$ the ellipses are larger for $\varepsilon>0$ and smaller for $\varepsilon<0$.

Thus the case $\omega_{1}=1.1$ with $\varepsilon>0$ is similar to the case $\omega_{1}=0.9$ for negative $\varepsilon$. On the other hand for $\varepsilon<0$ the ellipses become smaller (Fig. 14b) for increasing $|\varepsilon|$ and for $\varepsilon$ approaching the critical value $\varepsilon=-0.21598$ they tend to the $\mathrm{x}$-axis. 

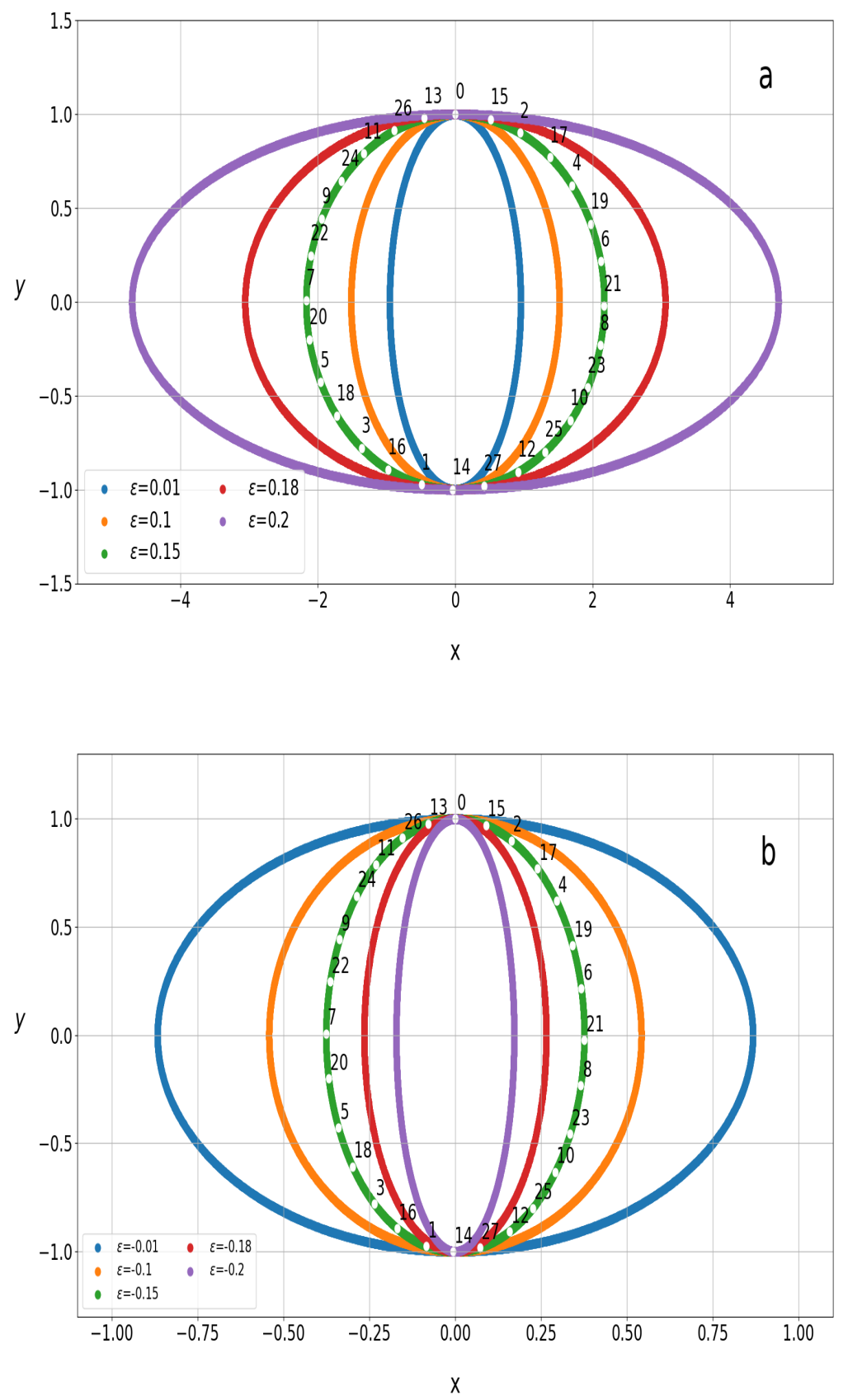

Figure 14: Invariant curves on the stroboscopic surface of section in the case $\omega=2, \omega_{1}=1.1, x_{0}=0, y_{0}=1$ for various values of $\varepsilon$ (a) for $\varepsilon>0$ the sizes of the curves increase as $\varepsilon$ increases and tend to infinity, as $\varepsilon$ tends to the critical value $\varepsilon_{\text {crit }}=0.21598$. (b) for $12<0$ the sizes of the curves decrease as $\varepsilon$ decreases and tend to a straight line from $y=-1$ to $y=1$ as $\varepsilon$ tends to the critical value $\varepsilon_{\text {crit }}=-0.21598$. 
In all cases the critical values of $\varepsilon$ for the same $\omega$ and $\omega_{1}$ are the same for positive and negative $\varepsilon$ but the limiting ellipse in one case (positive $\varepsilon$ for $\omega_{1}<1$, negative $\varepsilon$ for $\omega_{1}>1$ ) tend to the x-axis, while in the opposite cases the ellipse tends to infinity along the $\mathrm{x}$-axis.

\section{The integrals in a resonant case}

The theory of integrals in resonant cases, independent of time, has been developed in detail [28, 29]. However the applicability of the direct method of Contopoulos to resonant cases periodic in time has not yet been fully explored [29].

In the present case we will construct a formal integral in a particular resonant case of the Mathieu equation, namely in the case where the periodic term has a frequency $\omega$ which is double of the basic frequency $\omega_{1}$ of the unperturbed problem.

When $\omega_{1}=\omega / 2=1$ the series expansion of the integral $\Phi$ contains secular terms (namely time dependent terms which cannot be written in trigonometric form) and cannot be used. In this case it is appropriate to use as zeroth order solutions of the form $x=\frac{\sqrt{2 \Phi_{0}}}{\omega_{1}} \sin \left(\omega_{1}\left(t-t_{0}\right)\right), y=\sqrt{2 \Phi_{0}} \cos \left(\omega_{1}\left(t-t_{0}\right)\right)$ and find

$$
\begin{aligned}
\Phi_{1}= & \frac{1}{8}\left[\left(y^{2}-x^{2}\right) \cos (2 t)-2 x y \sin (2 t)-\left(y^{2}+x^{2}\right) \cos \left(2 t_{0}\right)\right] \\
& +\frac{1}{2}\left(y^{2}+x^{2}\right) \sin \left(2 t_{0}\right) t
\end{aligned}
$$

We observe that $\Phi_{1}$ contains the secular term

$$
\Phi_{1 s e c}=\frac{1}{2}\left(y^{2}+x^{2}\right) \sin \left(2 t_{0}\right) t
$$

However in this resonant case we can construct two more zero order integrals:

$$
\begin{aligned}
& C_{0}=2 \Phi_{0} \cos \left(\omega t-2 \omega_{1}\left(t-t_{0}\right)\right) \stackrel{\omega=2 \omega_{1}}{=} 2 \Phi_{0} \cos \left(2 \omega_{1} t_{0}\right) \\
& S_{0}=2 \Phi_{0} \sin \left(\omega t-2 \omega_{1}\left(t-t_{0}\right)\right) \stackrel{\omega=2 \omega_{1}}{=} 2 \Phi_{0} \sin \left(2 \omega_{1} t_{0}\right)
\end{aligned}
$$

We start by writing $C_{0}$ in the form

$$
C_{0}=\left(y^{2}-\omega_{1}^{2} x^{2}\right) \cos (\omega t)+2 \omega_{1} x y \sin (\omega t)
$$


which is periodic in time with period $T=\frac{2 \pi}{\omega}$. Then we calculate higher order terms in $C=C_{0}+\varepsilon C_{1}+\varepsilon^{2} C_{2}+\ldots$ In particular $C_{1}$ is

$$
C_{1}=\int_{0}^{t} \frac{\partial C_{0}}{\partial y} \frac{\partial H_{1}}{\partial x} d t
$$

and after some algebra we find

$$
\begin{aligned}
C_{1}=\frac{1}{2 \omega\left(\omega-\omega_{1}\right)}[ & y^{2}(1-\cos (2 \omega t))+x^{2}\left(\cos (2 \omega t)\left(2 \omega \omega_{1}-\omega_{1}^{2}\right)+\omega_{1}^{2}-2 \omega^{2}+2 \omega \omega_{1}\right) \\
& -2 \omega x y \sin (\omega t)]
\end{aligned}
$$

which does not have secular terms. Setting $\omega=2, \omega_{1}=1$ we have

$$
C_{1}=\frac{1}{4}\left[\left(y^{2}-3 x^{2}\right)(1-\cos (4 t))-4 x y \sin (4 t)\right]
$$

and for $t=k \pi$ we get $C_{1}=0$. If we calculate $C_{2}$ we find

$$
C_{2}=\int_{0}^{t} \frac{\partial C_{1}}{\partial y} \frac{\partial H_{1}}{\partial x} d t
$$

and for $\omega=2, \omega_{1}=1$ we find

$$
\begin{aligned}
C_{2}=\frac{1}{64} & {\left[y^{2}\left[-10 \cos (2 t)+\frac{5}{3} \cos (6 t)-13 \cos \left(2 t_{0}\right)+\frac{64}{3}\right]\right.} \\
& +x^{2}\left[-22 \cos (2 t)-\frac{37}{3} \cos (6 t)-13 \cos \left(2 t_{0}\right)+\frac{64}{3}\right] \\
& -2 x y[6 \sin (2 t)-5 \sin (6 t)]]-\frac{1}{8}\left(y^{2}+x^{2}\right) \sin \left(2 t_{0}\right) t
\end{aligned}
$$

Therefore $C_{2}$ contains the same secular term as $\Phi_{1}$ (up to a multiplicative constant) and we can combine the series of $\Phi$ and $C$ in order to avoid these terms. In particular if we multiply $\Phi$ by $\varepsilon q_{1}$ we find an integral

$$
\bar{C}=C_{0}+\varepsilon\left(q_{1} \Phi_{0}+C_{1}\right)+\varepsilon^{2}\left(q_{1} \Phi_{1}+C_{2}\right)+\ldots
$$

that does not have any secular term up to the order $\varepsilon^{2}$ if we take $q_{1}=\frac{1}{4}$. In a similar way we eliminate the secular terms of order $\varepsilon^{3}$ by adding an appropriate term $\varepsilon^{2} q_{2}\left(\Phi_{0}+\varepsilon \Phi_{1}+\ldots\right)$ and so on. 
The integral (37) describes a stroboscopic surface of section which for small $\varepsilon$ represents a hyperbola. Namely for $t=0$ or $t=k \pi$ we find

$$
\bar{C}_{s t r}=\left(y^{2}-x^{2}\right)+\frac{\varepsilon}{4}\left(x^{2}+y^{2}\right)+\ldots
$$

In Fig. 15 a we show the orbits corresponding to $\varepsilon=0.05$ and $\varepsilon=0.15$ and observe that the larger the $\varepsilon$ the faster the escape to infinity. In Fig. $15 \mathrm{~b}$ we draw their corresponding stroboscopic sections for times up to $t=15 \mathrm{~T}$ and see that they form two hyperbolae. Then we superimpose the hyperbolae predicted by the integral (38). We observe the very good agreement between the numerical results and the approximate integral of motion. In a similar way one may construct integrals of motion for every combination of $\omega, \omega_{1}$ that leads to resonances.
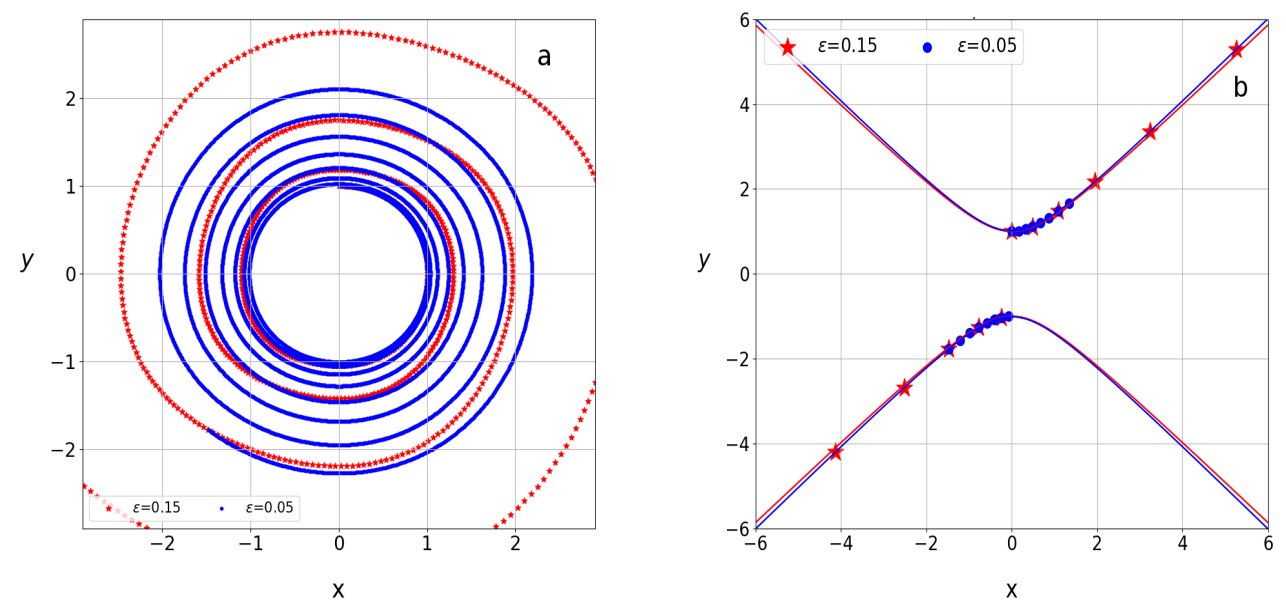

Figure 15: a) Two orbits in the resonant case $\omega=2, \omega_{1}=1$ for $\varepsilon=0.05$ (blue curve) and $\varepsilon=0.15$ (red curve). We observe the faster escape to infinity in the case of $\varepsilon=0.15 \mathrm{~b}$ ) The successive points (blue dots for $\varepsilon=0.05$ and red stars for $\varepsilon=0.15$ ) of two orbits on the stroboscopic surface of section lie, with a very good accuracy, on the correponding blue and red invariant curves given by Eq. (38). 


\section{Conclusions}

We studied the orbits and the stroboscopic invariant curves in the Hamiltonian $H=H_{0}+\varepsilon H_{1}$, where $H_{0}=\frac{1}{2}\left(\omega_{1}^{2} x^{2}+y^{2}\right)$ with $y=d x / d t$ and $H_{1}=-x^{2} \cos (\omega t)$. This Hamiltonian is equivalent to a Mathieu equation. We presented a method for constructing formal integrals of motion which are written as series expansions in $\varepsilon$ and converge if $|\varepsilon|$ is smaller than a critical value $\varepsilon_{\text {crit }}$. We studied in detail the forms of the orbits and of the integrals for various values of $\varepsilon, \omega$ and $\omega_{1}$. Our main results are the following:

1. The integral is quadratic in $x$ and $y$ of the form

$$
\Phi=C_{x} \omega_{1}^{2} x^{2}+C_{y} y^{2}+C_{x y} x y
$$

where $C_{x}, C_{y}, C_{x y}$ are series in $\varepsilon$. In non-resonant cases the integral forms invariant curves that are similar concentric ellipses on a stroboscopic Poincaré surface of section. The points of orbits with $t=$ $k T(k=1,2, \ldots, T=2 \pi / \omega)$ lie on such ellipses when the series converge.

2. We found the forms of the orbits that fill, in general, elliptical rings, leaving an empty region near the center $x=y=0$. We found also the distribution of the points on the stroboscopic surface of section.

3. For particular values of $\varepsilon$ the orbits are periodic.

4. For values of $\omega$ and $\omega_{1}$ below the resonance $\omega-2 \omega_{1}=0$ the stroboscopic ellipses become thinner along $x$ for $\varepsilon>0$, as $\varepsilon$ increases. As $\varepsilon \rightarrow \varepsilon_{\text {crit }}$ the ellipse tends to a straight line along the y-axis. For $\varepsilon>\varepsilon_{\text {crit }}$ the orbits spiral outwards and tend to infinity. On the other hand for $\varepsilon<0$ the ellipses become thinner along y as $\varepsilon$ decreases and as $\varepsilon \rightarrow-\varepsilon_{\text {crit }}$ they tend to a straight line along the $\mathrm{x}$-axis.

5. For any given set of physical parameters $\omega, \omega_{1}$ and initial conditions $x_{0}, y_{0}$, there are two escape values of $\varepsilon$, one positive and one negative, with the same absolute value $\left|\varepsilon_{\text {crit }}\right|$. We checked that these values are the same as given for the escape values of $q$ by other authors [21]. On

the other hand the orbits and the invariant curves are quite different for symmetric values of $\varepsilon$. 
6. The present system can be considered as a 2-d Hamiltonian system with two variables $x$ and $t$ and corresponding momenta $y$ and $E$ where $-E$ is the energy. If $|\varepsilon|<\varepsilon_{\text {crit }}$ the values of $E$ oscillate between a maximum and a minimum, while if $|\varepsilon|>\varepsilon_{\text {crit }}$ the values of $|E|$ extend to infinity.

7. If $\omega-2 \omega_{1}>0$ the orbits for $\varepsilon>0$ are of the opposite type from the orbits for $\omega-2 \omega_{1}<0$. Namely they are more elongated along $x$ as $\varepsilon$ increases for $\varepsilon>0$ and more elongated along $y$ as $\varepsilon$ decreases for $\varepsilon<0$.

8. In resonant cases the integrals are of different form and need to be constructed for every given resonance. We made an application in one resonant case where the invariant curves are hyperbolae and are very well approximated by a second approximate integral of motion.

Further work will refer to more general non-linear potentials where chaos becomes apparent.

\section{Acknowledgements}

This research was conducted in the framework of the program of the RCAAM of the Academy of Athens "Study of the dynamical evolution of the entanglement and coherence in quantum systems". The authors want to thank Dr. Christos Efthymiopoulos for his useful comments.

\section{References}

[1] G. Contopoulos, M. Moutsoulas, Resonance cases and small divisors in a third integral of motion, Astron. J. 70 (1965) 817.

[2] F. Gustavson, On constructing formal integrals of a hamiltonian system near an equilibrium point, Astron. J. 71 (1966) 670.

[3] A. Giorgilli, A computer program for integrals of motion, Comp. Phys. Comm. 16 (1979) 331.

[4] C. Efthymiopoulos, Z. Sándor, Optimized nekhoroshev stability estimates for the trojan asteroids with a symplectic mapping model of coorbital motion, Mon. Not. Roy. Astron. Soc. 364 (2005) 253. 
[5] G. Contopoulos, Adiabatic invariants and the "third" integral, J. Math. Phys. 7 (1966) 788.

[6] A. Markeyev, Third-order resonance in a hamiltonian system with one degree of freedom, J. App. Math. Mech. 58 (5) (1994) 793-804.

[7] A. Markeev, Stability of equilibrium states of hamiltonian systems: a method of investigation, Mech. Solids 39 (6) (2004) 1-8.

[8] A. Markeev, On a multiple resonance in linear hamiltonian systems, Dokl. Phys. 50 (5) (2005) 278-282.

[9] A. Markeev, Multiple parametric resonance in hamilton systems, J. App. Math. Mech. 70 (2) (2006) 176 - 194.

[10] A. P. Markeev, On the birkhoff transformation in the case of complete degeneracy of the quadratic part of the hamiltonian, Regul. Chaotic Dyn. 20 (3) (2015) 309-316.

[11] O. Kholostova, Non-linear oscillations of a hamiltonian system with one degree of freedom and fourth-order resonance, J. App. Math. Mech. 62 (6) (1998) 883-892.

[12] O. Kholostova, The periodic motions of a non-autonomous hamiltonian system with two degrees of freedom at parametric resonance of the fundamental type, J. App. Math. Mech. 66 (4) (2002) $529-538$.

[13] O. Kholostova, Resonant periodic motions of hamiltonian systems with one degree of freedom when the hamiltonian is degenerate, J. App. Math. Mech. 70 (4) (2006) 516-526.

[14] B. S. Bardin, V. Lanchares, On the stability of periodic hamiltonian systems with one degree of freedom in the case of degeneracy, Regul. Chaotic. Dyn. 20 (6) (2015) 627-648.

[15] A. D. Bruno, Normal form of a hamiltonian system with a periodic perturbation, Comput. Math. Math. Phys. 60 (1) (2020) 36-52.

[16] A. D. Bruno, Normalization of a periodic hamiltonian system, Program. Comput. Soft. 46 (2020) 76-83. 
[17] H. E. Kandrup, J. Drury, Chaos in cosmological hamiltonians, Ann. N. Y. Acad. Sci. 867 (1) (1998) 306.

[18] H. E. Kandrup, I. M. Vass, I. V. Sideris, Transient chaos and resonant phase mixing in violent relaxation, Mon. Not. Roy. Astron. Soc. 341 (2003) 927.

[19] B. Terzić, H. E. Kandrup, Orbital structure in oscillating galactic potentials, Mon. Not. Roy. Astron. Soc. 347 (2004) 957.

[20] C. Efthymiopoulos, G. Contopoulos, Chaos in bohmian quantum mechanics, J. Phys. A 39 (2006) 1819.

[21] N. W. McLachlan, Theory and application of Mathieu functions, Oxford University Press, 1951.

[22] J. Richards, The Mathieu equation, in: Analysis of Periodically TimeVarying Systems, Springer, 1983, p. 93.

[23] É. Mathieu, Mémoire sur le mouvement vibratoire d'une membrane de forme elliptique., J. Math. Pure. Appl. 13 (1868) 137.

[24] M. Leibscher, B. Schmidt, Quantum dynamics of a plane pendulum, Phys. Rev. A 80 (2009) 012510.

[25] T. Birkandan, M. Hortacsu, Examples of heun and mathieu functions as solutions of wave equations in curved spaces, J. Phys. A 40 (2007) 1105.

[26] J. K. Fink, Physical Chemistry in depth, Springer, 2009.

[27] L. Ruby, Applications of the Mathieu equation, Amer. J. Phys. 64 (1996) 39.

[28] G. Contopoulos, Resonance cases and small divisors in a third integral of motion. 1., Astron. J. 68 (1963) 763.

[29] G. Contopoulos, Order and Chaos in Dynamical Astronomy, Springer, 2002. 